\title{
Detection of the Fermi/GBT pulsar PSR J2032+4127 with the EVN and the DiFX correlator in Bonn
}

\author{
Javier Moldón*, Marc Ribó, Josep M. Paredes \\ Departament d'Astronomia i Meteorologia, Institut de Ciències del Cosmos (ICC), Universitat \\ de Barcelona (IEEC-UB) \\ Martí $i$ Franquès, 1 \\ 08028 Barcelona, Spain \\ E-mail: Gmoldondam.ub.es, mribodam.ub.es, Gmparedesdub.edu
}

\section{Bosch-Ramon}

Dublin Institute for Advanced Studies

E-mail: valentiecp.dias.ie

\section{Kramer}

Jodrell Bank Centre for Astrophysics

Max Planck Institut für Radioastronomie

E-mail: mkramerempifr-bonn.mpq.de

\section{A.G. Lyne}

Jodrell Bank Centre for Astrophysics

E-mail: andrew. Iynedmanchester.ac.uk

\section{B.W. Stappers}

Jodrell Bank Centre for Astrophysics

E-mail: ben.stappersdmanchester.ac.uk

We observed the Fermi/GBT pulsar PSR J2032+4127 with the EVN at $1.6 \mathrm{GHz}$ in March 2010. The source has a period-averaged flux density of $0.18 \mathrm{mJy}$ and a duty cycle of approximately $10 \%$ at this frequency. To obtain a good position of the source, we observed it using phase referencing with $6 \mathrm{EVN}$ antennas at $1 \mathrm{Gbps}$, and we correlated the data using pulsar gating in the new DiFX correlator in Bonn. A preliminary data reduction shows that the faint pulsar was detected with a signal-to-noise ratio of 8 , with an improvement of a factor of 2 thanks to the use of pulsar gating. This has provided the first accurate VLBI position of this radio pulsar. Future EVN observations will allow us to obtain an accurate proper motion of PSR J2032+4127, which will shed light to the possible association of this pulsar with the unidentified very-high-energy gamma-ray source TeV J2032+4130.

10th European VLBI Network Symposium and EVN Users Meeting: VLBI and the new generation of radio arrays

September 20-24, 2010

Manchester Uk

\footnotetext{
*Speaker.
} 


\section{Introduction}

Pulsars have usually been discovered after performing blind searches in the radio domain. In some cases, pulsed emission above $\mathrm{GeV}$ energies has been detected, either by the MAGIC groundbased Cherenkov telescope (Aliu et al. 2008) or by the Fermi satellite (Abdo et al. 2009). A blind search of pulsars with the Fermi satellite lead to the discovery of the 143 ms period gamma-ray pulsar PSR J2032+4127, which falls in the region of the unidentified very-high-energy source $\mathrm{TeV}$ J2032+4130. This pulsar was further studied in the radio domain using the Green Bank Telescope, which provided a detection with a period-averaged flux density is $0.12 \pm 0.03 \mathrm{mJy}$ at $2 \mathrm{GHz}$ and $0.65 \pm 0.13 \mathrm{mJy}$ at $0.8 \mathrm{GHz}$ (Camilo et al. 2009). The dispersion measure was of $114.8 \pm 0.1 \mathrm{pc} \mathrm{cm}^{-3}$, corresponding to a distance of $3.6 \mathrm{kpc}$ according to the Cordes \& Lazio (2002) model.

\section{Pulsar Gating}

VLBI observations of radio pulsars can provide accurate pro-per motions, and in some cases parallaxes (Brisken et al. 2002). In normal VLBI observations of radio pulsars one measures the period-averaged flux density along the integration time, although most of the time the pulsar is not emitting. The instantaneous flux density during the pulsation is usually tens of times higher than the period-averaged value, because the duty cycle (the ratio of the pulse width to the pulse period) is usually below $10 \%$. To optimize VLBI observations of pulsars one can take advantage

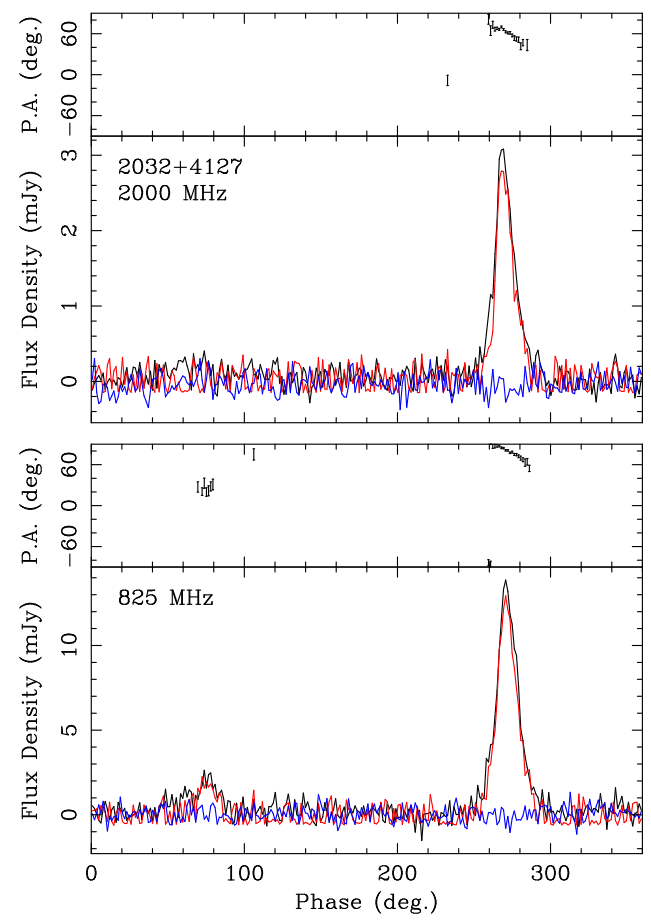

Figure 1: Polarimetric profiles of PSR J2032+4127 based on GBT observations at 2.0 and $0.8 \mathrm{GHz}$. The black traces correspond to the total intensity, while the red and blue lines correspond, respectively, to linear and circular polarization. From Camilo et al. (2009). 
of this fact by only correlating the data during on-pulse, and disabling correlation during off-pulse. This technique is known as pulsar gating. The total correlated time is considerably reduced (a few minutes for every observed hour), increasing the final image noise by a factor proportional to one over the square root of the duty cycle. However, the signal increase is approximately proportional to one over the duty cycle. This provides a theoretical signal-to-noise ratio increase proportional to one over the square root of the duty cycle of the pulsar. Typically, the real signal-to-noise improves a factor between 2 and 6, which can make the difference between a non-detection and a clear detection, which leads to accurate astrometry. More sophisticated correlations can be used, as weighting the signal according to the pulse shape (matched filter).

Until now it has not been possible to perform pulsar gating correlation of EVN data in Europe, and therefore it was difficult to combine the high sensitivity of this array with the enhancement of pulsar gating. Pulsar gating correlations can be naturally set in the new software DiFX correlators, and have been successfully performed in the VLBA and LBA correlators. For the EVN, the recently updated DiFX correlator in Bonn can now be used to correlate data using pulsar gating. This possibility will also be available soon at the new SFXC software EVN correlator at JIVE.

\section{Observations and results}

We observed the Fermi/GBT pulsar PSR J2032+4127 with the EVN at $1.6 \mathrm{GHz}$ in March 2010. The source has a period-averaged flux density of $0.18 \mathrm{mJy}$ and a duty cycle of approximately $10 \%$ at this frequency. To obtain a good position of the source, we observed it using phase referencing with $6 \mathrm{EVN}$ antennas (Ef, Jb, $\mathrm{Mc}$, $\mathrm{On}, \mathrm{Tr}$, and $\mathrm{Wb}$ ) at $1 \mathrm{Gbps}$, and we correlated the data using pulsar gating in the new DiFX correlator in Bonn. The updated pulsar ephemerides needed to set the gates were obtained through regular pulsar observations at Jodrell Bank. All the technical support required for correlating and checking the EVN data was provided by Walter Alef, David Graham, John Morgan, and Helge Rottmann (Max-Planck-Institut für Radioastronomie). A preliminary data reduction shows that the faint pulsar was detected with a signal-to-noise ratio of 8 , with an improvement of a factor of 2 thanks to the use of pulsar gating. This has provided the first accurate VLBI position of this faint radio pulsar. Future EVN observations will allow us to obtain an accurate proper motion of PSR J2032+4127, which will shed light to the possible association of this pulsar with the unidentified very high energy gamma-ray source TeV J2032+4130.

\section{References}

[1] Abdo, A. A., et al. 2009, Science, 325, 840

[2] Aliu, E., et al. 2008, Science, 322, 1221

[3] Brisken, W. F., Benson, J. M., Goss, W. M., \& Thorsett, S. E. 2002, ApJ, 571, 906

[4] Camilo, F., et al. 2009, ApJ, 705, 1

[5] Cordes, J.M., \& Lazio, T.J.W., arXiv:astro-ph/0207156 
ON-PULSE

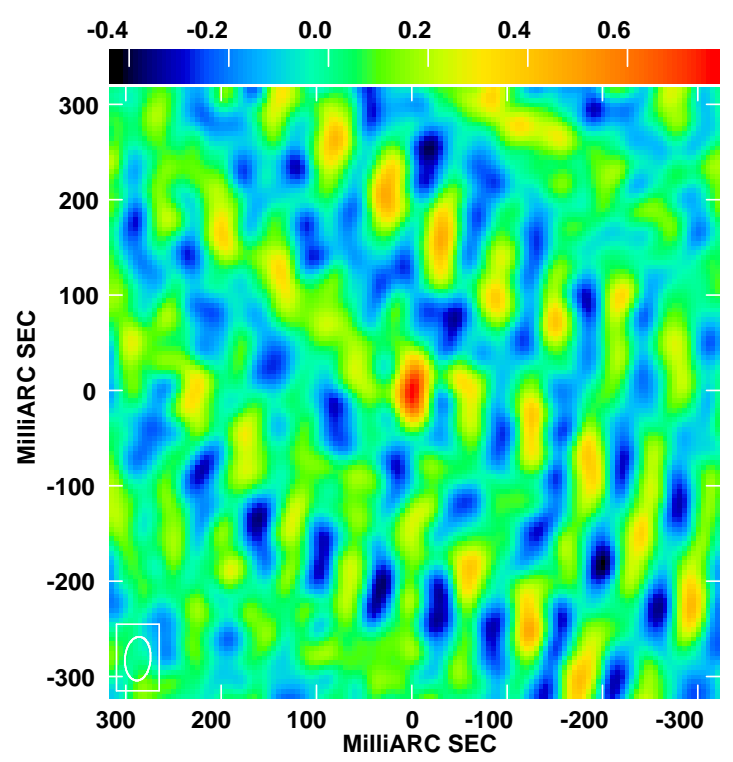

OFF-PULSE

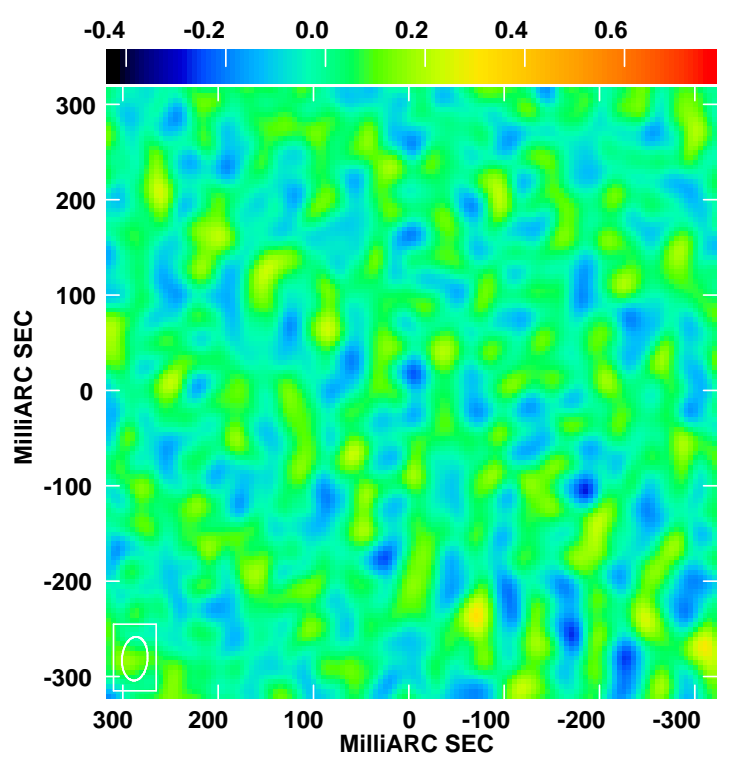

Figure 2: Preliminary images of PSR J2032+4127 obtained with 6 stations of the EVN at 1.6 GHz. The data were correlated with the new DiFX correlator in Bonn using pulsar gating, which improved the signalto-noise ratio by a factor of 2 . The left image corresponds to the data correlated during on-pulse $(0.1$ phase bin), and the right image during off-pulse (for a 0.1 phase bin as well). 